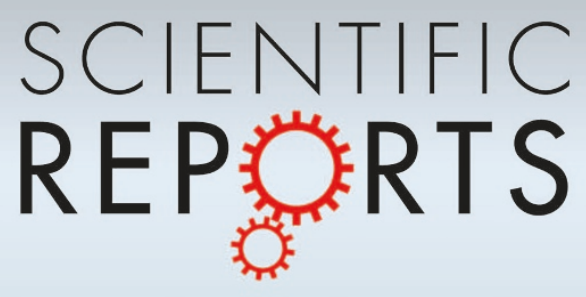

\title{
OPEN A facile process for soak-and-peel \\ AND DEVICES delamination of CVD graphene from substrates using water
}

SUBJECT AREAS:

ELECTRONIC PROPERTIES

SYNTHESIS OF GRAPHENE

Received

15 October 2013

Accepted

8 January 2014

Published

24 January 2014

Correspondence and requests for materials should be addressed to

Priti Gupta ${ }^{1 *}$, Pratiksha D. Dongare 1,2*, Sameer Grover' , Sudipta Dubey' , Hitesh Mamgain ${ }^{3}$, Arnab Bhattacharya' \& Mandar M. Deshmukh'

${ }^{1}$ Tata Institute of Fundamental Research, Homi Bhabha Road, Colaba, Mumbai 400005, India, ${ }^{2}$ Birla Institute of Technology and Science, Vidyavihar Campus, Pilani, Rajasthan 333031, India, ${ }^{3}$ WITec GmbH, Lise-Meitner-Str. 6, D-8908 1 Ulm, Germany.

We demonstrate a simple technique to transfer chemical vapour deposited (CVD) graphene from copper and platinum substrates using a soak-and-peel delamination technique utilizing only hot deionized water. The lack of chemical etchants results in cleaner CVD graphene films minimizing unintentional doping, as confirmed by Raman and electrical measurements. The process allows the reuse of substrates and hence can enable the use of oriented substrates for growth of higher quality graphene, and is an inherently inexpensive and scalable process for large-area production.

M.M.D. (deshmukh@

M.M.D. (deshmukh@
tifr.res.in)

* These authors contributed equally to

this work.

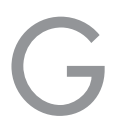

raphene, a monolayer honeycomb lattice structure of $\mathrm{sp}^{2}$-bonded carbon atoms, has become a subject of great interest due to its extraordinary optical, mechanical, and electronic properties ${ }^{1-3}$. Successful isolation of graphene by the mechanical exfoliation of highly oriented pyrolytic graphite (HOPG) has opened doors for new innovations in the field of nanoelectronics ${ }^{4-6}$. Since then many new methods have emerged to synthesize and isolate single to few-layer graphene ${ }^{3}$ especially on large area substrates. These methods include reduction of graphite oxide ${ }^{7}$, ultrasonication of graphite ${ }^{8}$, synthesis on $\mathrm{SiC}$ substrate ${ }^{9,10}$, and chemical vapour deposition (CVD) on metal substrates such as $\mathrm{Ni}^{11}, \mathrm{Cu}^{12,13}, \mathrm{Ru}^{14}$ and $\mathrm{Pt}^{15,16}$.

Of all these methods, the low-pressure growth of graphene on $\mathrm{Cu}$ foils, in particular, is known to be advantageous in terms of controlled graphene size, number of layers and quality ${ }^{12}$. It has also been shown that better quality graphene can be grown on $\mathrm{Cu}(111)$ oriented grains ${ }^{17}$. Thus CVD graphene growth on Cu produces large areas of mostly monolayer graphene and is a promising way of producing large area graphene for practical nanoelectronics applications $s^{3,18}$. To fully realize the advantages of the CVD graphene growth there must be a reliable method for transferring the graphene from metallic $\mathrm{Cu}$ substrates to more useful substrates like insulating substrates $^{19}$, flexible/stretchable substrate ${ }^{20}$, and transparent electrodes ${ }^{21,22}$. Pt (111), as a substrate for CVD graphene growth, is also interesting because it has minimum effect on the physical properties of graphene due to its very weak graphene-substrate interaction. Further, it has been shown that the electronic structure of the graphene grown on Pt is nearly the same as that of the free standing graphene ${ }^{23}$. In addition, Pt does not get oxidized easily like other metal substrates such as $\mathrm{Cu}$.

Currently the processes used to transfer large-area and high-quality graphene synthesized on metal substrates require wet etching of the metal substrates ${ }^{12,24}$. These processes trap ionic species between graphene and substrate interface which act as scattering centers and lead to degradation of the electrical properties of the devices fabricated on the graphene. Further, the etching process also results in loss of metal ultimately increasing the cost of the transfer process; this is especially true for precious metals and oriented single crystal substrates that are expensive. Electrochemical methods to transfer graphene without metal loss have been demonstrated but they involve chemicals like $\mathrm{NaOH}$ and are complex ${ }^{25,26}$. An intercalation method to transfer graphene from $\mathrm{Pt}$ to other substrates has also been shown, but for small size graphene flakes only ${ }^{27}$. To overcome these problems, we demonstrate a novel facile method to transfer graphene from metal substrates $(\mathrm{Cu}$ and $\mathrm{Pt}$ ) with hot deionized (DI) water without using any chemical etchants. This results in transferred graphene layers that are clean and show improved properties compared to graphene layers transferred using the conventional etching route.

DI water has potential use in transfer processes due to its capability to penetrate nanoscale hydrophobichydrophilic interfaces and separate them. Such methods have been used to selectively position ${ }^{21}$ and transfer ${ }^{28}$ graphene flakes and other nanostructures using difference in affinity to water. In our method, we first coat the 
graphene layer with poly(methyl methacrylate) (PMMA) as a support material. PMMA has been used to support and transfer mechanically exfoliated graphene flakes ${ }^{29,30}$ and CVD-grown graphene to target substrates ${ }^{19}$. We then exploit the differential interaction of water with the hydrophobic graphene $e^{31,32}$ and the hydrophilic metal like $\mathrm{Cu}^{33}$ or $\mathrm{Pt}^{34}$ to delaminate the graphene from the substrate used for CVD growth.

The most important difference in our DI water Soak-and-Peel Delamination (SPeeD) method and presently established methods to transfer graphene from $\mathrm{Cu}^{12,20,22,24,29,30,35-37}$ and $\mathrm{Pt}$ substrates ${ }^{26}$ is that, our SPeeD method is very simple since it does not involve use of any chemical etchants and hence provides cleaner graphene. Additionally the metal substrate $(\mathrm{Cu}$ and $\mathrm{Pt})$ can be reused - an aspect desirable for industrial production. As our technique utilizes the difference in the interaction of the graphene and substrates with water, this method can be extended to a larger class of CVD substrates for a variety of applications. The SPeeD technique uses only DI water, hence contamination due to ionic species can be significantly reduced ensuring that the electrical properties are not degraded as typically seen for graphene transferred via processes using chemical etchants to remove the $\mathrm{Cu}$ substrate.

\section{Results}

CVD graphene was grown both in continuous ${ }^{12}$ and island growth ${ }^{38}$ modes on $\mathrm{Cu}$ and $\mathrm{Pt}$ substrates (details about the growth provided in Supplementary Section I). The SPeeD transfer process is schematically depicted in Figure 1. After the CVD growth of graphene on $\mathrm{Cu}$ foil (Figure 1(a)), we spin-coated the graphene with PMMA (310 nm thick resist (Microchem EL-9) at a speed of $3200 \mathrm{rpm}$ for $45 \mathrm{~s}$ ) followed by baking for 7 minutes at $175^{\circ} \mathrm{C}$ (Figure 1(b)). To avoid crumpling of the resist after delamination and for easier handling, Kapton tape (3M 5413) is stuck on the resist and uniform pressure is applied using a teflon roller (Figure 1(c)).
The $\mathrm{Cu}$ foil, with the tape attached, is then immersed in a beaker of DI water maintained at $90^{\circ} \mathrm{C}$ for 2 hours (Figure $1(\mathrm{~d})$ ). During this period DI water penetrates the graphene-Cu interface. Subsequently, the Kapton tape (to which the PMMA/graphene stack is attached) is slowly peeled away with tweezers leaving behind the $\mathrm{Cu}$ foil (see Supplementary Movie S1 which shows the key steps of our SPeeD process). The target substrate, a $300 \mathrm{~nm}$ thick $\mathrm{SiO}_{2}$-coated p-type silicon wafer, was cleaned using oxygen plasma reactive ion etching to ensure better adhesion between graphene and the substrate. The Kapton tape, clamped to a glass slide, and the target substrate are brought into contact and heated for 40 minutes at $140^{\circ} \mathrm{C}$ (Figure 1(e)). After allowing the sample to cool for 20 minutes, the Kapton tape is detached from the glass slide and the stack is put in acetone to remove the EL-9 layer. Subsequently, rapid thermal annealing (RTA) $\left(300^{\circ} \mathrm{C}\right.$ for 10 minutes and $350^{\circ} \mathrm{C}$ for 5 minutes in $100 \mathrm{sccm} \mathrm{Ar}$ ) is done to remove any residual PMMA (Figure 1(f)), thus completing the transfer process. The success rate of our transfer process is about 90 percent (for statistics of the samples transferred by SPeeD method, see Supplementary Section II).

We used the DI water based SPeeD method to transfer graphene grown on Pt foils as well. With our technique, this can be easily done without curling of graphene and without using any chemical like $\mathrm{NaOH}$ that can lead to unintentional doping of graphene ${ }^{26}$. The only difference from the process for releasing used for graphene on $\mathrm{Cu}$ is that a thicker resist layer was used for $\mathrm{Pt}$ compared to $\mathrm{Cu}$ and the resist was not baked. The remaining procedure for transfer of graphene from $\mathrm{Pt}$ is same as that for graphene on $\mathrm{Cu}$.

To benchmark the quality of graphene transferred using our SPeeD process, we transferred two graphene samples, grown on $\mathrm{Cu}$ foils from the same batch and in the same graphene growth run, by two different transfer methods. Though the quality of our CVD graphene is not as good as the best reported in the literature $^{12,19,39}$, the comparison of graphene samples grown in the same

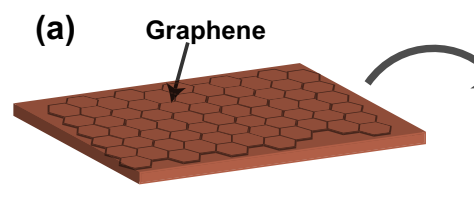

(b)

(c)

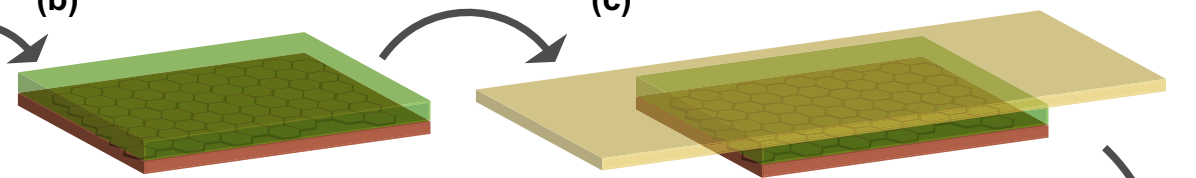

(d)

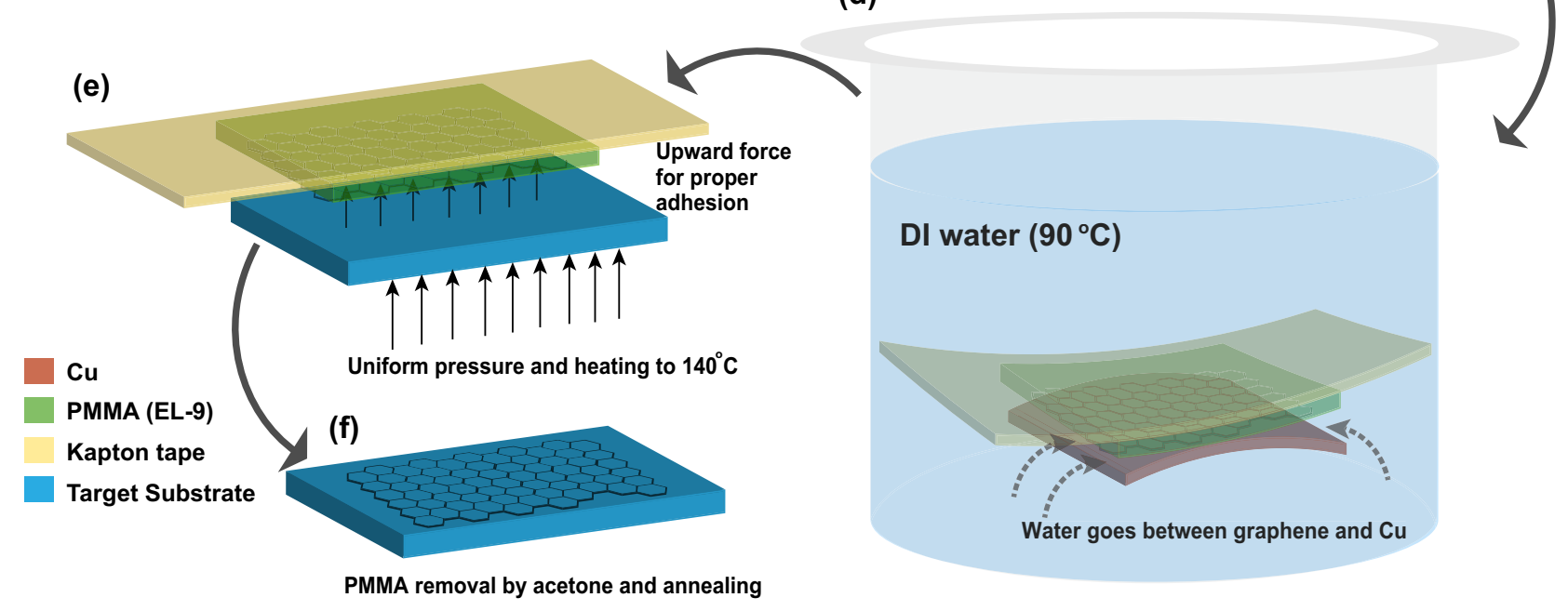

Figure 1 Schematic showing the steps of the DI water SPeeD graphene transfer method. (a) CVD graphene is grown on Cu substrate. (b) PMMA is spin-coated on the graphene grown on $\mathrm{Cu} / \mathrm{Pt}$ substrate. (c) Kapton tape is pressed on PMMA with a teflon roller. (d) The stack is immersed in DI water at $90^{\circ} \mathrm{C}$. Water penetrates between graphene and Cu substrate and separates them. (e) Kapton tape with PMMA and graphene on it is pressed against the target substrate and heated for 40 minutes at $140^{\circ} \mathrm{C}$. (f) The PMMA on the target substrate is removed with acetone and RTA. 

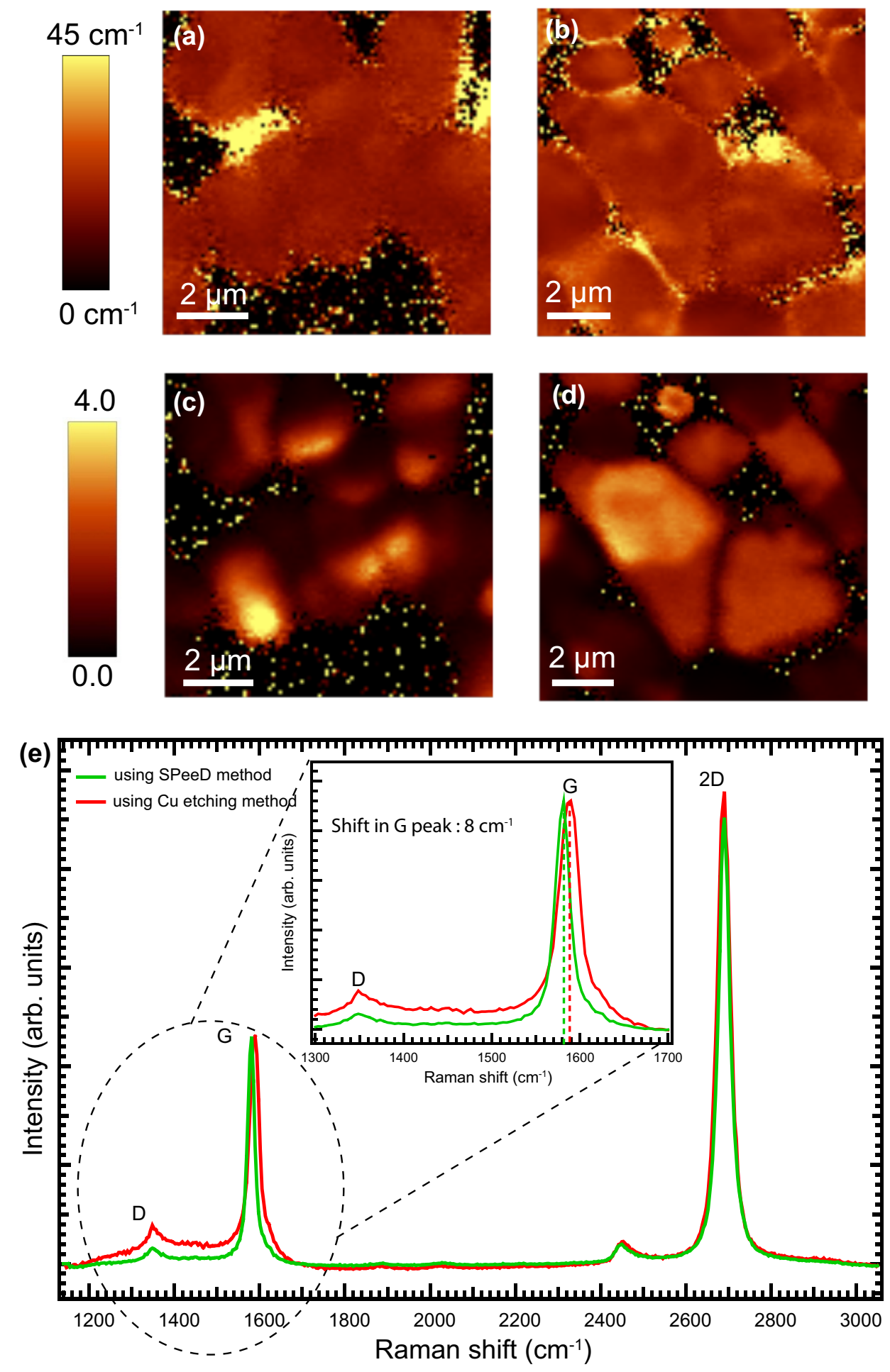

Figure 2 Comparative Raman spectroscopy of graphene transferred by etching $\mathrm{Cu}$ to release graphene and by using the SPeeD process. Integrated Raman mapping over an area of $9 \mu \mathrm{m} \times 9 \mu \mathrm{m}$ for the FWHM of the G peak of graphene transferred by (a) our SPeeD method using DI water, and (b) conventional etching of $\mathrm{Cu}$ by ammonium persulphate solution. Colourscale maps of $2 \mathrm{D} / \mathrm{G}$ peak intensity ratio for same area of graphene transferred by (c) our SPeeD method and (d) conventional Cu etching method. (e) Comparison of the spatially averaged Raman scattering spectra of graphene transferred by the two different methods. Inset of (e) shows the blue shift of $8 \mathrm{~cm}^{-1}$ in the G peak for graphene transferred by Cu etching which indicates that it is p-type doped.

run under identical conditions but transferred by different processes should show the influence of the transfer process on the defect level, doping level and quality of graphene. The first sample was transferred onto a $\mathrm{SiO}_{2}$-coated $\mathrm{Si}$ substrate using conventional etching of $\mathrm{Cu}$ with ammonium persulphate solution (details in Supplementary Section
III). The second sample was transferred to an identical substrate using our SPeeD method with DI water without using any etchant. We compare the two samples transferred using Raman spectroscopy measurements ${ }^{40}$ and electrical transport measurements. The results of the comparison are discussed in the following sections. 
(a)

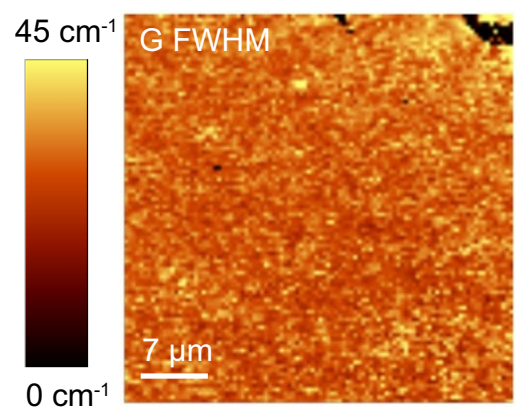

(b)

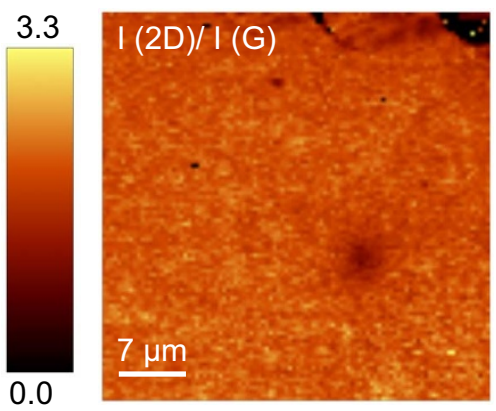

Figure 3 Raman measurement data for the CVD graphene grown on Pt and transferred using SPeeD method. Colourscale maps of (a) the width of the G peak (b) $2 \mathrm{D} / \mathrm{G}$ peak intensity ratio across an area $40 \mu \mathrm{m} \times 40 \mu \mathrm{m}$ of graphene grown on Pt.

\section{Discussion}

Confocal Raman spectroscopy measurements were performed on both the samples using a WITec Alpha 300R confocal Raman microscope. Figures 2(a) and (b) compare the Raman maps of the full width at half maximum (FWHM) of the G peak for the two graphene samples transferred by the two different methods. Figures 2(c) and (d) compare colourscale maps of $2 \mathrm{D} / \mathrm{G}$ peak intensity ratio for same area of graphene transferred by two different methods. The spatiallyaveraged Raman spectra of graphene over a $9 \mu \mathrm{m} \times 9 \mu \mathrm{m}$ area for both samples are shown in Figure 2(e). To evaluate shift in peak positions, the spectra are aligned with reference to the $\mathrm{Si}$ substrate peak $\left(520 \mathrm{~cm}^{-1}\right)$, and to compare relative intensity of the various features, the spectra are intensity normalized to the graphene $G$ peak value. The ratio of the intensity of the $2 \mathrm{D}$ peak to $\mathrm{G}$ peak in both the samples is comparable and has the value of $\sim 2$, which indicates that the graphene is monolayer ${ }^{40}$.

The interesting observation is that the integrated Raman spectrum of the graphene transferred by the SPeeD method has a lower D peak intensity than that of the sample transferred by conventional $\mathrm{Cu}$ etching (Figure 2(e)). This suggests that the graphene transferred by the SPeeD process has less defects compared to the one transferred by conventional $\mathrm{Cu}$ etching ${ }^{41}$. Further, the inset (Figure 2(e)) shows that the FWHM of the G peak of the graphene transferred by the SPeeD process is narrower than that of the graphene transferred by
$\mathrm{Cu}$ etching. This points to a lower disorder in the SPeeD transferred sample $^{42}$. Additionally, the $\mathrm{G}$ peak is blue shifted ${ }^{43}$ in the graphene transferred by $\mathrm{Cu}$ etching. This indicates that the graphene transferred by $\mathrm{Cu}$ etching is $\mathrm{p}$-type doped compared to the other sam$\mathrm{ple}^{40,43}$. This $\mathrm{p}$-type doping can be attributed to charge impurities present in the $\mathrm{Cu}$ etchant or the presence of defects in the graphene. Thus, transferring graphene using our SPeeD method without any use of $\mathrm{Cu}$ etchant reduces the probability of graphene getting doped by ionic impurities. Transport measurements on two graphene samples (discussed later) grown using the same recipe but different transfer methods corroborate this observation about reduced doping in the SPeeD transferred samples.

The SPeeD method has been successfully applied to transfer CVD graphene grown on Pt foils as well. Raman measurements on the CVD graphene transferred via the SPeeD method from Pt foils to $300 \mathrm{~nm} \mathrm{SiO}{ }_{2}$-coated p-doped $\mathrm{Si}$ are shown in Figure 3.

Furthermore, the reusability of $\mathrm{Cu}$ and $\mathrm{Pt}$ substrates using our SPeeD method was investigated. A Raman spectroscopy comparison of CVD graphene grown using a recipe for continuous growth of fewlayer graphene on fresh and reused $\mathrm{Cu}$ foils, showed that the quality of graphene does not degrade on reusing the metal substrate (for details, see Supplementary Section IV).

The Dirac point for graphene transferred by $\mathrm{Cu}$ etching is shifted to the high positive gate voltage region $(>50 \mathrm{~V}$ ) (Figure $4(\mathrm{~b})$ ) indi-
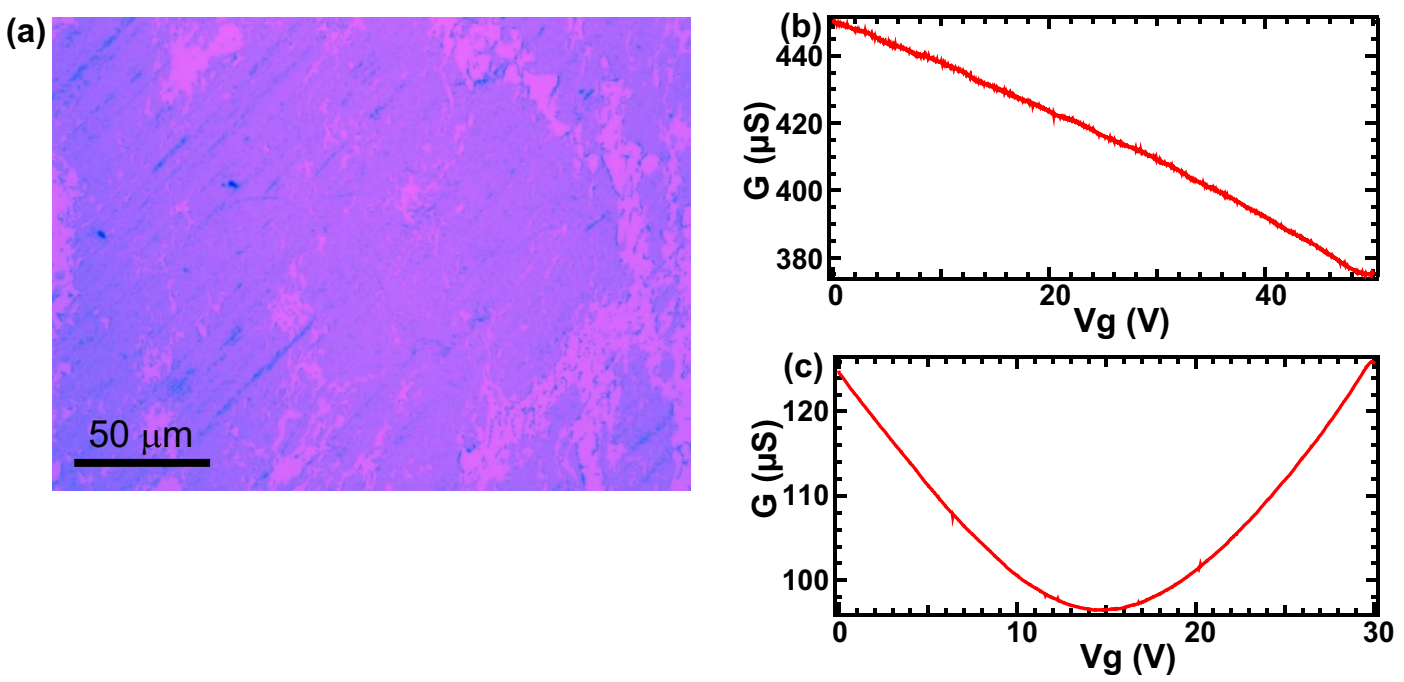

Figure 4 Electrical transport measurements for devices fabricated using graphene samples transferred by two different methods. (a) Optical image of CVD graphene grown on Cu transferred by SPeeD method. (b) Gating curve for the device fabricated with graphene transferred by conventional Cu etching method. (c) Gating curve for device fabricated with graphene transferred by SPeeD method. The source-drain spacing of the devices used for measurements was $\sim 5 \mu \mathrm{m}$. 

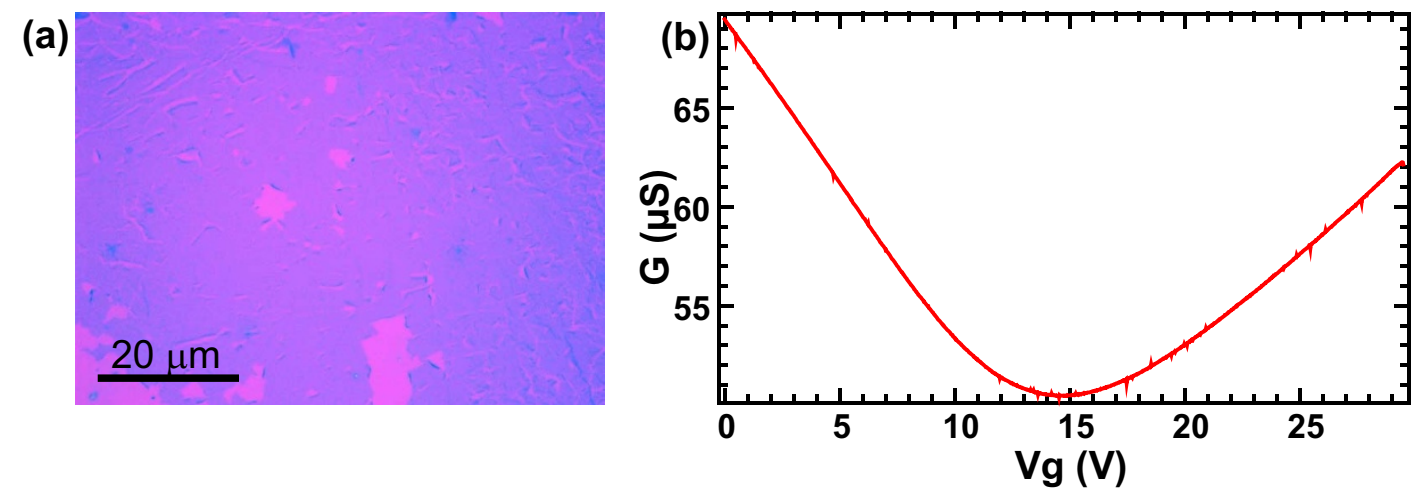

Figure 5 | Electrical transport measurement for device fabricated using graphene sample transferred from Pt substrate. (a) Optical image of the graphene transferred by SPeeD from Pt substrate to $300 \mathrm{~nm} \mathrm{SiO}{ }_{2}$-coated p-type doped Si substrate. (b) Transport measurement for the device fabricated on the graphene transferred from Pt. The source-drain spacing of the device used for measurement was $\sim 7 \mu \mathrm{m}$.

cating that it is highly p-type doped which also agrees well with the Raman measurements. The Dirac point is observable in DI water transferred graphene at $\sim 15 \mathrm{~V}$ (Figure $4(\mathrm{c})$ ) indicating that the sample is much cleaner in comparison. The electrical transport measurements for the devices fabricated using graphene samples transferred by two different methods were done in air at room temperature. Prior to measurement the samples were annealed at $350^{\circ} \mathrm{C}$ in $100 \mathrm{sccm}$ forming gas for $20 \mathrm{~min}$.

An optical image of the graphene transferred from Pt substrate by SPeeD method on $300 \mathrm{~nm} \mathrm{SiO}$-coated -type doped silicon target substrate is shown in Figure 5(a). The gating curve for the device fabricated using our transfer method shows the presence of the Dirac peak at $\sim 15 \mathrm{~V}$ (Figure $5(\mathrm{~b})$ ) once again indicating a relatively clean sample.

The Raman and electrical data unequivocally demonstrate that the SPeeD method using hot DI water is an effective method to transfer CVD graphene. We observe that the delamination of freshly grown CVD graphene layers is more difficult than the delamination of graphene that has been exposed to air for a few days. This allows us to propose a possible mechanism for the efficacy of the SPeeD method. Our observations suggest that diffusion of oxygen at the interface of graphene and copper plays an important role ${ }^{44}$. The intercalation of oxygen modifies the nature of interaction between graphene and substrate leading to a wicking effect where a thin layer of water separates graphene and substrate. This process is expedited on heating in DI water. In addition, the presence of nanometer scale holes in the CVD graphene greatly accelerates the delamination process ${ }^{45}$ by providing several pathways for delamination. This process is analogous to the previous reports ${ }^{46,47}$ where the buffered HF $\mathrm{SiO}_{2}$ etch rate along the interface of $\mathrm{SiO}_{2}$ and graphene is significantly enhanced due to a wicking effect.

In summary, we have successfully demonstrated a novel and simple Soak-and-Peel Delamination method using DI water to transfer CVD-grown graphene from metal substrates like copper and platinum to other substrates of interest. This method does not expose graphene to any harsh chemicals and hence ensures that electrical properties of graphene are not affected. This method is cost effective because no etchant is used and since the metal is not etched it can also be recycled many times reducing large scale production costs. It will also allow the use of single crystals of $\mathrm{Cu}$ (111) for improved growth without consuming the copper single crystals. This simple technique demonstrates low cost, clean transfer of graphene and opens doors for its widespread use. The SPeeD process may also provide a generic route to exploit differential hydrophilic/hydrophobic interactions to delaminate other 2D layered materials from grown substrates.

1. Geim, A. K. \& Novoselov, K. S. The rise of graphene. Nature Mater. 6, 183-191 (2007).
2. Neto, A. H. C., Guinea, F., Peres, N. M. R., Novoselov, K. S. \& Geim, A. K. The electronic properties of graphene. Rev. Mod. Phys. 81, 109-162 (2009).

3. Novoselov, K. S. et al. A roadmap for graphene. Nature 490, 192-200 (2012).

4. Novoselov, K. S. et al. Electric field effect in atomically thin carbon films. Science 306, 666-669 (2004)

5. Zhang, Y., Small, J. P., Pontius, W. V. \& Kim, P. Fabrication and electric-fielddependent transport measurements of mesoscopic graphite devices. Appl. Phys. Lett. 86, 073104 (2005).

6. Wang, L. et al. One-dimensional electrical contact to a two-dimensional material. Science 342, 614-617 (2013).

7. Stankovich, S. et al. Synthesis of graphene-based nanosheets via chemical reduction of exfoliated graphite oxide. Carbon 45, 1558-1565 (2007)

8. Hernandez, Y. et al. High-yield production of graphene by liquid-phase exfoliation of graphite. Nature Nanotech. 3, 563-568 (2008).

9. Berger, C. et al. Ultrathin epitaxial graphite: $2 \mathrm{D}$ electron gas properties and a route toward graphene-based nanoelectronics. J. Phys. Chem. B 108, 19912-19916 (2004).

10. Kim, J. et al. Layer-resolved graphene transfer via engineered strain layers. Science 342, 833-836 (2013).

11. Yu, Q., Lian, J., Siriponglert, S., Li, H., Chen, Y. P. \& Pei, S.-S. Graphene segregated on Ni surfaces and transferred to insulators. Appl. Phys. Lett. 93, 113103 (2008).

12. Li, X. et al. Large-area synthesis of high-quality and uniform graphene films on copper foils. Science 324, 1312-1314 (2009).

13. Hao, Y. et al. The role of surface oxygen in the growth of large single-crystal graphene on copper. Science 342, 720-723 (2013).

14. Sutter, P. W., Flege, J.-I. \& Sutter, E. A. Epitaxial graphene on ruthenium. Nature Mater. 7, 406-411 (2008).

15. Kang, B. J., Mun, J. H., Hwang, C. Y. \& Cho, B. J. Monolayer graphene growth on sputtered thin film platinum. J. Appl. Phys. 106, 104309 (2009).

16. Sutter, P., Sadowski, J. T. \& Sutter, E. Graphene on Pt (111): Growth and substrate interaction. Phys. Rev. B 80, 245411 (2009).

17. Wood, J. D., Schmucker, S. W., Lyons, A. S., Pop, E. \& Lyding, J. W. Effects of polycrystalline $\mathrm{Cu}$ substrate on graphene growth by chemical vapor deposition. Nano Lett. 11, 4547-4554 (2011).

18. Geim, A. K. Graphene: status and prospects. Science 324, 1530-1534 (2009).

19. Reina, A. et al. Large area, few-layer graphene films on arbitrary substrates by chemical vapor deposition. Nano Lett. 9, 30-35 (2009).

20. Kim, K. S. et al. Large-scale pattern growth of graphene films for stretchable transparent electrodes. Nature 457, 706-710 (2009).

21. Bonaccorso, F., Sun, Z., Hasan, T. \& Ferrari, A. C. Graphene photonics and optoelectronics. Nature Photon. 4, 611-622 (2010).

22. Bae, S. et al. Roll-to-roll production of 30 -inch graphene films for transparent electrodes. Nature Nanotech. 5, 574-578 (2010).

23. Gao, M. et al. Epitaxial growth and structural property of graphene on $\mathrm{Pt}$ (111). Appl. Phys. Lett. 98, 033101 (2011).

24. Li, X. et al. Transfer of large-area graphene films for high-performance transparent conductive electrodes. Nano Lett. 9, 4359-4363 (2009).

25. Wang, Y. et al. Electrochemical delamination of CVD-grown graphene film: toward the recyclable use of copper catalyst. ACS Nano 5, 9927-9933 (2011)

26. Gao, L. et al. Repeated growth and bubbling transfer of graphene with millimetresize single-crystal grains using platinum. Nat. Commun. 3, 699 (2012).

27. Ma, D. et al. Clean transfer of graphene on Pt foils mediated by a carbon monoxide intercalation process. Nano Res. 6, 671-678 (2013).

28. Schneider, G. F., Calado, V. E., Zandbergen, H., Vandersypen, L. M. K. \& Dekker, C. Wedging transfer of nanostructures. Nano Lett. 10, 1912-1916 (2010).

29. Bonaccorso, F. et al. Production and processing of graphene and $2 \mathrm{~d}$ crystals. Mater. Today 15, 564-589 (2012).

30. Reina, A. et al. Transferring and identification of single- and few-layer graphene on arbitrary substrates. J. Phys. Chem. C 112, 17741-17744 (2008). 
31. Bianco, V., Iskrov, S. \& Franzese, G. Understanding the role of hydrogen bonds in water dynamics and protein stability. J. Biol. Phys. 38, 27-48 (2012).

32. Wang, S., Zhang, Y., Abidi, N. \& Cabrales, L. Wettability and surface free energy of graphene films. Langmuir 25, 11078-11081 (2009).

33. Valatte, G. Hydrophilicity of metal surfaces: silver, gold and copper electrodes. J. Electroanal. Chem. Interfacial Electrochem. 139, 285-301 (1982).

34. Gardner, J. R. \& Woods, R. The hydrophilic nature of gold and platinum. J. Electroanal. Chem. Interfacial Electrochem. 81, 285-290 (1977).

35. Levendorf, M. P., Ruiz-Vargas, C. S., Garg, S. \& Park, J. Transfer-free batch fabrication of single layer graphene transistors. Nano Lett. 9, 4479-4483 (2009).

36. Lee, Y. et al. Wafer-scale synthesis and transfer of graphene films. Nano Lett. 10, 490-493 (2010).

37. Regan, W. et al. A direct transfer of layer-area graphene. Appl. Phys. Lett. 96 , 113102 (2010).

38. Han, Z. et al. Suppression of multilayer graphene patches during CVD graphene growth on copper. arXiv: 1205.1337 (2012).

39. Li, X. et al. Large-area graphene single crystals grown by low-pressure chemical vapor deposition of methane on copper. J. Am. Chem. Soc. 133, 2816-2819 (2011).

40. Ferrari, A. C. \& Basko, D. M. Raman spectroscopy as a versatile tool for studying the properties of graphene. Nature Nanotech. 8, 235-246 (2013).

41. Ferrari, A. C. Raman spectroscopy of graphene and graphite: disorder, electronphonon coupling, doping and nonadiabatic effects. Solid State Commun. 143, 47-57 (2007).

42. Cançado, L. G. et al. Quantifying defects in graphene via Raman spectroscopy at different excitation energies. Nano Lett. 11, 3190-3196 (2011).

43. Das, A. et al. Monitoring dopants by Raman scattering in an electrochemically top-gated graphene transistor. Nature Nanotech. 3, 210-215 (2008).

44. Kidambi, P. R. et al. Observing graphene grow: catalyst-graphene interactions during scalable graphene growth on polycrystalline copper. Nano Lett. 13, 4769-4778 (2013).

45. O'Hern, S. C. et al. Selective molecular transport through intrinsic defects in a single layer of cvd graphene. ACS Nano 6, 10130-10138 (2012).
46. Bolotin, K. I. et al. Ultrahigh electron mobility in suspended graphene. Solid State Commun. 146, 351-355 (2008).

47. Du, X., Skachko, I., Barker, A. \& Andrei, E. Y. Approaching ballistic transport in suspended graphene. Nature Nanotech. 3, 491-495 (2008).

\section{Acknowledgments}

The work at TIFR was supported by the Government of India. M.M.D. acknowledges the support of the Department of Science and Technology, India, through the Swarnajayanti fellowship.

\section{Author contributions}

P.G. and P.D.D. contributed equally to this work. They developed the process, performed the measurements, analyzed the data, and wrote the manuscript. S.G. and S.D. contributed to the device fabrication and measurements. H.M. helped with Raman measurements. A.B. and M.M.D. planned and supervised the study. All authors discussed the results and commented on the manuscript.

\section{Additional information}

Supplementary information accompanies this paper at http://www.nature.com/ scientificreports

Competing financial interests: The authors declare no competing financial interests. How to cite this article: Gupta, P. et al. A facile process for soak-and-peel delamination of CVD graphene from substrates using water. Sci. Rep. 4, 3882; DOI:10.1038/srep03882 (2014).

(i) $\Theta$ This work is licensed under a Creative Commons AttributionNonCommercial-NoDerivs 3.0 Unported license. To view a copy of this license, visit http://creativecommons.org/licenses/by-nc-nd/3.0 